\title{
Some Remarks on Betti Numbers of Random Polygon Spaces
}

\author{
Clément Dombry, ${ }^{1}$ Christian Mazza ${ }^{2}$ \\ ${ }^{1}$ Laboratoire de Mathématiques et Applications, Téléport 2- BP30179, Boulevard \\ Pierre et Marie Curie, 86962 Futuroscope Chasseneuil Cedex, France; \\ e-mail: clement.dombry@math.univ-poitiers.fr \\ ${ }^{2}$ Département de Mathématique, Université de Fribourg, Chemin du Musée 23, \\ CH-1700 Fribourg, Suisse; e-mail: Christian.Mazza@unifr.ch
}

\begin{abstract}
Polygon spaces such as $M_{\ell}=\left\{\left(u_{1}, \ldots, u_{n}\right) \in S^{1} \times \ldots S^{1}, \sum_{i=1}^{n} l_{i} u_{i}=0\right\} / S O(2)$, or the three-dimensional analogs $N_{\ell}$ play an important rôle in geometry and topology, and are also of interest in robotics where the $l_{i}$ model the lengths of robot arms. When $n$ is large, one can assume that each $l_{i}$ is a positive real valued random variable, leading to a random manifold. The complexity of such manifolds can be approached by computing Betti numbers, the Euler characteristics, or the related Poincaré polynomial. We study the average values of Betti numbers of dimension $p_{n}$ when $p_{n} \rightarrow \infty$ as $n \rightarrow \infty$. We also focus on the limiting mean Poincaré polynomial, in two and three dimensions. We show that in two dimensions, the mean total Betti number behaves as the total Betti number associated with the equilateral manifold where $l_{i} \equiv \bar{l}$. In three dimensions, these two quantities are not any more asymptotically equivalent. We also provide asymptotics for the Poincaré polynomials. @ 2009 Wiley Periodicals, Inc. Random Struct. Alg., 37, 67-84, 2010

Keywords: configuration space; Betti number; poincaré polynomial; random polygonal linkage; random manifold
\end{abstract}

\section{INTRODUCTION}

\subsection{Background}

In this note, we consider a question raised by Farber in [2]. We study closed planar $n$-gons whose sides have fixed lengths $l_{1}, \ldots, l_{n}$ where $l_{i}>0$ for $1 \leq i \leq n$. The set of polygonal linkage in $\mathbb{R}^{2}$ 


$$
M_{\ell}=\left\{\left(u_{1}, \ldots, u_{n}\right) \in S^{1} \times \ldots S^{1} ; \sum_{i=1}^{n} l_{i} u_{i}=0\right\} / S O(2)
$$

parametrizes the variety of all possible shapes of such planar $n$-gons with sides given by $\ell=\left(l_{1}, \ldots, l_{n}\right)$. The unit vector $u_{i} \in \mathbb{C}$ indicates the direction of the $i$-th side of the polygon. The condition $\sum l_{i} u_{i}=0$ expresses the property of the polygon being closed. The rotation group $S O(2)$ acts on the set of side directions $\left(u_{1}, \ldots, u_{n}\right)$ diagonally.

Polygon spaces play a fundamental rôle in topology and geometry, as illustrated for example by Kempe Theorem which states that "Toute courbe algébrique peut être tracée à l'aide d'un système articulê", see e.g. [9]. In [3], the author provides other examples of such universality results in topology. Polygon spaces generated an active research area in geometry (see e.g. [5], [6], or [10]), but are also of strong interest in applications like robotics where each $l_{i}$ models the length or a robot arm (see e.g. [2], [3], [4], and [5]). We can also point out potential applications in polymer science where such polygons model proteins. In systems composed of a large number $n \gg 1$ of components, the $l_{i}$ are usually only partially known, so that we can assume that each $l_{i} \in \mathbb{R}^{+}$is random. We denote by $\mu_{n}$ the distribution of $\ell$. We will obtain our results under the following assumption:

(H) $\mu_{n}$ is a product measure $\mu_{n}=\mu^{\otimes n}$ with $\mu$ a diffuse measure on $(0, \infty)$ such that

$$
\int e^{\eta x} \mu(\mathrm{d} x)<\infty \quad \text { for some } \eta>0 .
$$

The measure is said diffuse if it satisfies $\mu(\{x\})=0$ for all $x \in \mathbb{R}$. Notice that $M_{t \ell}$ and $M_{\ell}$ are equal when $t>0$, so that the measure $\mu_{n}$ might be seen as a probability measure on the unit simplex $\triangle^{n-1}$.

To get some idea on the nature of the random manifold $M_{\ell}$, one can study the stochastic behavior of invariants like Betti numbers, the Euler characteristics, or the total Betti number (see below). Here, we focus on the Betti numbers $b_{p}\left(M_{\ell}\right)$, for dimensions $p=p_{n}$ growing with $n$. We recall the result of [5] describing Betti numbers of planar polygon spaces as functions of the length vector $\ell$. In what follows, $[n]$ denotes the set $\{1, \ldots, n\}$. A subset $J \subset[n]$ is called short if

$$
\sum_{j \in J} l_{j}<\sum_{j \notin J} l_{j} .
$$

It is called median if $\sum_{j \in J} l_{j}=\sum_{j \notin J} l_{j}$. Let $1 \leq i_{0} \leq n$ be such that $l_{i_{0}}$ is maximal among $l_{1}, \ldots, l_{n}$. Denote by $a_{p}(\ell)$ the number of short subsets $J \subset[n]$ of cardinality $|J|=p+1$ and containing $i_{0}$. Denote by $\tilde{a}_{p}(\ell)$ the number of median subsets $J \subset[n]$ of cardinality $|J|=p+1$ and containing $i_{0}$. Then one has for $p=0,1, \ldots, n-3$

$$
b_{p}\left(M_{\ell}\right)=a_{p}(\ell)+\tilde{a}_{p}(\ell)+a_{n-3-p}(\ell),
$$

so that the Poincare polynomial of the random manifold is given by

$$
p_{M_{\ell}}(t)=\sum_{p=0}^{n-3} b_{p}\left(M_{\ell}\right) t^{p}=q(t)+t^{n-3} q\left(\frac{1}{t}\right)+r(t),
$$

where

$$
q(t)=\sum_{k=0}^{n-3} a_{k} t^{k} \text { and } r(t)=\sum_{k=0}^{n-3} \tilde{a}_{k} t^{k}
$$


see [2]. The total Betti number $B\left(M_{\ell}\right)$ defined by

$$
B\left(M_{\ell}\right)=\sum_{p=0}^{n-3} b_{p}\left(M_{\ell}\right)=p_{M_{\ell}}(1)
$$

provides ideas on the size or on the complexity of the manifold $M_{\ell}$. We will study the asymptotic behavior of $B\left(M_{\ell}\right)$ when $n$ is large and $\ell$ is random. We first give some examples following [5].

In the equilateral case where each $l_{i}$ is equal to some $\bar{l}>0$, it turns out that one can give exact formulas for the various Betti numbers, and therefore for $B\left(M_{\bar{\ell}}\right)$ : assume $n=2 r+1$ odd. Then $b_{k}\left(M_{\bar{\ell}}\right)=\left(\begin{array}{c}n-1 \\ k\end{array}\right)$ when $k<r-1, b_{k}\left(M_{\bar{\ell}}\right)=2\left(\begin{array}{c}n-1 \\ r-1\end{array}\right)$ when $k=r-1$ and $b_{k}\left(M_{\bar{\ell}}\right)=\left(\begin{array}{c}n-1 \\ k+2\end{array}\right)$ when $k>r-1$. The related total Betti number is then given by $p_{M_{\bar{\ell}}}(1)=2^{n-1}-\left(\begin{array}{c}n-1 \\ r\end{array}\right)$. For arbitrary large $n$, one has (see [5])

$$
p_{M_{\bar{\ell}}}(1)=B_{n}=2^{n-1}\left(1-\sqrt{\frac{2}{\pi n}}+o\left(n^{-1 / 2}\right)\right), \quad n \rightarrow \infty .
$$

For pentagons, that is when $n=5$, the moduli space $M_{\ell}$ is a compact orientable surface of genus not exceeding 4.

The vector length $\ell$ is said to be generic when $\sum_{i=1}^{n} l_{i} \varepsilon_{i} \neq 0$, for any $\varepsilon=\left(\varepsilon_{i}\right)_{1 \leq i \leq n}$, where, $\forall i \varepsilon_{i} \in\{-1,+1\}$. When $n$ is even, equilateral weights with $l_{i} \equiv \bar{l}$ are not generic. In [5] the authors proved that for generic $\ell$, the total Betti number $B\left(M_{\ell}\right)$ is bounded by $2 B_{n-1}$, so that the explicit formulas obtained for equilateral $n$-gons provide bounds for the maximum over $\ell$ of $B\left(M_{\ell}\right)$.

\subsection{Results}

In [4] the authors considered the special case where $\mu$ is the uniform probability measure on the unit interval with $\mu_{n}=\mu^{\otimes n}$, and the case where $\mu_{n}$ is the uniform measure on the simplex $\Delta^{n-1}$. It was proven that for fixed $p \geq 0$, the average $p$-dimensional betti number

$$
\mu_{n}\left[b_{p}\left(M_{\ell}\right)\right]=\int b_{p}\left(M_{\ell}\right) \mu_{n}(\mathrm{~d} \ell)
$$

is asymptotically equivalent to $\left(\begin{array}{c}n-1 \\ p\end{array}\right)$, the difference going to zero at an exponential speed. The techniques use exact formulas for the volume of the intersection of a half space with a simplex. We will avoid such formulas to treat general diffuse probability measures using probabilistic techniques, since in fact such volume formulas do not exist for arbitrary measures. Next, both planar and spatial polygon spaces were considered in [2], and similar results for the moments of Betti numbers of fixed dimension $p$ were proved under an admissibility condition on $\mu_{n}$. As an open question, the author raises the issue of computing the average total Betti number

$$
\mu_{n}\left[B\left(M_{\ell}\right)\right]=\int B\left(M_{\ell}\right) \mu_{n}(\mathrm{~d} \ell) .
$$

We will consider more generally the mean Poincaré polynomial

$$
\bar{p}_{M_{\ell}}(t)=\mu_{n}\left[p_{M_{\ell}}(t)\right]=\int p_{M_{\ell}}(t) \mu_{n}(\mathrm{~d} \ell),
$$


with $\bar{p}_{M_{\ell}}(1)=\mu_{n}\left[B\left(M_{\ell}\right)\right]$. As the author notices, the knowledge of the individual average Betti numbers $\mu_{n}\left[b_{p}\left(M_{\ell}\right)\right]$ for large $n$ and fixed $p$ cannot help since the terms cannot simply be added up. We will therefore consider the asymptotic behavior of high-dimensional Betti numbers $\mu_{n}\left[b_{p_{n}}\left(M_{\ell}\right)\right]$, where $p_{n}$ goes to infinity when $n \rightarrow \infty$ (see Proposition 3.1).

We will obtain our results for product measure $\mu_{n}$ satisfying assumption (H) and assume throughout the article that this hypothesis is satisfied. We prove in Proposition 4.1 that the mean total Betti number is such that

$$
\bar{p}_{M_{\ell}}(1)=\mu_{n}\left[B\left(M_{\ell}\right)\right] \sim 2^{n-1}
$$

This shows that equilateral polygons (see (3)) are representative of the emerging average manifold as $n \gg 1$, as suggested in [2]. We will also consider the mean Poincaré polynomial as $n$ is large, and show that

$$
\bar{p}_{M_{\ell}}(t) \sim(1+t)^{n-1} \text { when } 0<t<1,
$$

and that

$$
\bar{p}_{M_{\ell}}(t) \sim(1+t)^{n-1} t^{-2} \text { when } t>1 \text {. }
$$

Further moments are also considered and their asymptotic is given in Proposition 4.3.

We next consider spatial polygon spaces

$$
N_{\ell}=\left\{\left(u_{1}, \ldots, u_{n}\right) \in S^{2} \times \ldots S^{2} ; \sum_{i=1}^{n} l_{i} u_{i}=0\right\} / S O(3) .
$$

It was proved in [7] that in this case, for generic length vector $\ell$, the even Betti numbers are given by

$$
b_{2 p}\left(N_{\ell}\right)=\sum_{j=0}^{p}\left(\hat{a}_{j}(\ell)-\hat{a}_{n-j-2}(\ell)\right)
$$

where $\hat{a}_{j}(\ell)$ denotes the number of short subsets $J \subset[n]$ of cardinality $|J|=j+1$ containing $n$. The Betti number of odd dimensions vanish. The related Poincaré polynomial is then given by

$$
p_{N_{\ell}}(t)=\frac{1}{1-t^{2}}\left(\sum_{J \in \mathcal{S}_{n}} t^{2(|J|-1)}-t^{2(n-|J|-1)}\right),
$$

where $J \in \mathcal{S}_{n}$ if and only if $\{n\} \subset J \subset[n]$ and $J$ is median or short. If $\ell$ is generic, there is no median set and this is equivalent to

$$
p_{N_{\ell}}(t)=\frac{1}{1-t^{2}} \sum_{j=0}^{n-1} \hat{a}_{j}\left(t^{2 j}-t^{2(n-j-2)}\right)=\frac{1}{1-t^{2}}\left[\hat{q}\left(t^{2}\right)-t^{2(n-2)} \hat{q}\left(t^{-2}\right)\right],
$$

where

$$
\hat{q}(t)=\sum_{j=0}^{n-1} \hat{a}_{j} t^{j}
$$


In the equilateral case where $l_{i} \equiv \bar{l},[8]$ proved that the $2 p$-dimensional Betti number $b_{2 p}\left(N_{\bar{\ell}}\right)$ is given by

$$
b_{2 p}\left(N_{\bar{\ell}}\right)=\sum_{i=0}^{p}\left(\begin{array}{c}
n-1 \\
i
\end{array}\right) \text {, }
$$

when $n=2 k+1 \geq 3$, so that the Euler characteristics or total Betti number is explicitely given as

$$
p_{N_{\bar{\ell}}}(1)=\sum_{i=0}^{k-1}\left(\begin{array}{c}
2 k \\
i
\end{array}\right)(k-i),
$$

with

$$
p_{N_{\bar{\ell}}}(1) \sim \sqrt{\frac{n}{2 \pi}} 2^{n-2}
$$

We will study the asymptotic behavior of the mean Poincaré polynomial

$$
\bar{p}_{N_{\ell}}(t)=\mu_{n}\left[p_{N_{\ell}}(t)\right]=\int p_{N_{\ell}}(t) \mu_{n}(\mathrm{~d} \ell)
$$

in the large $n$ limit by providing large deviations estimates. We will see that

$$
\bar{p}_{N_{\ell}}(1)=\mu_{n}\left[B\left(N_{\ell}\right)\right] \sim n 2^{n-2} \gg p_{N_{\bar{\ell}}}(1) .
$$

Furthermore, we will see in Proposition 4.2 that the mean Poincaré polynomial exhibits asymptotically a singular behavior in the neighborhood of $t=1$, that is

$$
\bar{p}_{N_{\ell}}(t) \sim \frac{\left(1+t^{2}\right)^{n-1}}{\left(1-t^{2}\right)} \quad \text { when } 0<t<1
$$

and

$$
\bar{p}_{N_{\ell}}(t) \sim \frac{\left(1+t^{2}\right)^{n-1}}{\left(t^{2}-1\right) t^{2}} \quad \text { when } t>1
$$

This shows that equilateral configuration spaces are not representative of the random manifold in dimension 3 when $n$ is large.

\section{PRELIMINARIES}

We introduce here the main technical tool used in our analysis of the Betti numbers of random polygon spaces: a probabilistic interpretation of formulas (1) and (5) in terms of random permutations and stopping times. We first introduce some notations.

For any length vector $\ell \in(0, \infty)^{n}$, we define $\tilde{\ell}$ obtained from $\ell$ by the following permutation of the coordinates : let $i_{0}$ be the minimal index such that $l_{i_{0}}$ is maximal among the $l_{i}, 1 \leq i \leq n$, and define $\tilde{\ell}=\left(\tilde{l}_{1}, \ldots, \tilde{l}_{n}\right)$ by $\tilde{l}_{n}=l_{i_{0}}, \tilde{l}_{i_{0}}=l_{n}$, and $\tilde{l}_{i}=l_{i}$ if $i \notin\left\{i_{0}, n\right\}$. 
We denote by $\sigma$ a random permutation of $\Sigma_{n-1}$ with uniform distribution $\mathcal{U}_{\Sigma_{n-1}}$. The stopping time $\tau_{\sigma}(\ell)$ is defined by

$$
\tau_{\sigma}(\ell)=\min \left\{0 \leq t \leq n-1 ; \sum_{i=1}^{t} l_{\sigma(i)}+l_{n}-\sum_{i=t+1}^{n-1} l_{\sigma(i)} \geq 0\right\} .
$$

We use also the notation $\tau(\ell)=\tau_{I d}(\ell)$ and $\tilde{\tau}(\ell)=\tau_{I d}(\tilde{\ell})$. Please note that these stopping times are well defined and that $\tau \leq n-1$ and $\tilde{\tau} \leq n-2$.

We denote by $k$ a random variable with binomial distribution $\mathcal{B}_{n-1, q}$ with parameters $n-1$ and $q \in[0,1]$.

First consider the planar case.

Lemma 2.1. The number $a_{p}(\ell)$ of short sets is given by

$$
a_{p}(\ell)=\left(\begin{array}{c}
n-1 \\
p
\end{array}\right) \mathcal{U}_{\Sigma_{n-1}}\left[\tau_{\sigma}(\tilde{\ell})>p\right]
$$

The number of median sets $\tilde{a}_{p}(\ell)$ vanishes $\mu_{n}$-almost surely.

Hence, the planar Betti numbers are given $\mu_{n}$-almost surely by

$$
b_{p}\left(M_{\ell}\right)=\mathcal{U}_{\Sigma_{n-1}}\left[\left(\begin{array}{c}
n-1 \\
p
\end{array}\right) \mathbf{1}_{\left\{\tau_{\sigma}(\tilde{\ell})>p\right\}}+\left(\begin{array}{c}
n-1 \\
p+2
\end{array}\right) \mathbf{1}_{\left\{\tau_{\sigma}(\tilde{\ell})>n-p-3\right\}}\right]
$$

and have expected value

$$
\mu_{n}\left[b_{p}\left(M_{\ell}\right)\right]=\mu_{n}\left[\left(\begin{array}{c}
n-1 \\
p
\end{array}\right) \mathbf{1}_{\{\tilde{\tau}(\ell)>p\}}+\left(\begin{array}{c}
n-1 \\
p+2
\end{array}\right) \mathbf{1}_{\{\tilde{\tau}(\ell)>n-p-3\}}\right]
$$

In the spatial case, the following representation holds.

Lemma 2.2. The coefficients $\hat{a}_{p}$ are given by

$$
\hat{a}_{p}(\ell)=\left(\begin{array}{c}
n-1 \\
p
\end{array}\right) \mathcal{U}_{\Sigma_{n-1}}\left[\tau_{\sigma}(\ell)>p\right]
$$

Hence, the even spatial Betti numbers are given $\mu_{n}$-almost surely by

$$
b_{2 p}\left(N_{\ell}\right)=2^{n-1}\left(\mathcal{U}_{\Sigma_{n-1}} \otimes B_{n-1,1 / 2}\right)\left[\mathbf{1}_{\left\{\tau_{\sigma}(\ell)>k ; 0 \leq k \leq p\right\}}-\mathbf{1}_{\left\{\tau_{\sigma}(\ell)>k ; n-p-2 \leq k \leq n-2\right\}}\right]
$$

and have expected value

$$
\mu_{n}\left[b_{2 p}\left(N_{\ell}\right)\right]=2^{n-1}\left(\mu_{n} \otimes B_{n-1,1 / 2}\right)\left[\mathbf{1}_{\{\tau(\ell)>k ; 0 \leq k \leq p\}}-\mathbf{1}_{\{\tau(\ell)>k ; n-p-2 \leq k \leq n-2\}}\right]
$$

Proof of Lemmas 2.1 and 2.2. We consider the planar case and prove the first lemma. The second lemma corresponding to the spatial case is proved with a very similar analysis.

From the definition of the coefficient $a_{p}(\ell)$, we have

$$
a_{p}(\ell)=\sum_{J \subset[n-1] ;|J|=p} \mathbf{1}_{A_{J}}(\tilde{\ell})
$$


where $A_{J}=\left\{\ell ; \sum_{j \in J} l_{j}+l_{n}-\sum_{j \notin J} l_{j}<0\right\}$. From the definition of $\tau_{\sigma}$, it is easily seen that $\ell \in A_{\{\sigma(1), \ldots, \sigma(p)\}}$ if and only if $\tau_{\sigma}(\ell)>p$. Furthermore, for each subset $J \subset[n-1]$ such that $|J|=p$, there are $p !(n-1-p)$ ! permutations $\sigma \in \Sigma_{n-1}$ such that $J=\{\sigma(1), \ldots, \sigma(p)\}$. As a consequence, the coefficient $a_{p}(\ell)$ rewrites

$$
a_{p}(\ell)=\frac{1}{p !(n-1-p) !} \sum_{\sigma \in \Sigma_{n-1}} \mathbf{1}_{\left\{\tau_{\sigma}(\tilde{\ell})>p\right\}}=\left(\begin{array}{c}
n-1 \\
p
\end{array}\right) \mathcal{U}_{\Sigma_{n-1}}\left[\tau_{\sigma}(\tilde{\ell})>p\right]
$$

From the definition of the coefficient $\tilde{a}_{p}(\ell)$, we have

$$
\tilde{a}_{p}(\ell)=\sum_{J \subset[n-1] ;|J|=p} \mathbf{1}_{B_{J}}(\tilde{\ell})
$$

where $B_{J}=\left\{\ell ; \sum_{j \in J} l_{j}+l_{n}-\sum_{j \notin J} l_{j}=0\right\}$. But it is easily seen that since $\mu$ is diffuse, the $\operatorname{sum} \sum_{j \in J} l_{j}+l_{n}-\sum_{j \notin J} l_{j}$ is also diffuse and $\mu_{n}\left(B_{J}\right)=0$ for any $J \subset[n-1]$. Hence $\tilde{a}_{p}(\ell)$ is almost surely equal to zero.

The formula for the Betti number $b_{p}(\ell)$ is then a reformulation of Eq. (1). Thanks to the invariance of $\mu_{n}$ under the action of the permutation group, the distribution of $\tau_{\sigma}(\tilde{\ell})$ under $\mu_{n}$ does not depend on $\sigma \in \Sigma_{n-1}$ and hence is equal to the distribution of $\tilde{\tau}$. The result for the expected value $\mu_{n}\left[b_{p}\left(M_{\ell}\right)\right]$ follows.

As will be clear in the sequel, the asymptotic behavior of the Betti numbers is strongly linked with the asymptotic behavior of the random variables $\tau(\ell)$ and $\tilde{\tau}(\ell)$. This is the point of the following lemma.

Lemma 2.3. The following weak convergences hold under $\mu_{n}$ as $n \rightarrow \infty$ :

1. weak law of large numbers:

$$
n^{-1} \tau \Rightarrow \delta_{1 / 2}
$$

2. central limit theorem:

$$
n^{-1 / 2}\left(\tau-\frac{n}{2}\right) \Rightarrow \mathcal{N}\left(0, \sigma_{\tau}^{2}\right)
$$

where $\sigma_{\tau}=\frac{\sigma}{2 m}, m=\mathbf{E}(l)$ and $\sigma^{2}=\operatorname{Var}(l)$.

3. large deviations: for any $\varepsilon>0$,

$$
\lim \sup n^{-1} \log \mu_{n}\left(\left|n^{-1} \tau-1 / 2\right| \geq \varepsilon\right)<0
$$

The same results also hold for $\tilde{\tau}$ instead of $\tau$ with the same variance $\sigma_{\tilde{\tau}}=\sigma_{\tau}$.

The proof is postponed to the appendix. 


\section{HIGH-DIMENSIONAL BETTI NUMBERS}

\subsection{Planar Polygons}

The following proposition gives the asymptotic of average high-dimensional Betti numbers. We say that two sequences of real numbers $\left(a_{n}\right)_{n \geq 0}$ and $\left(a_{n}\right)_{n \geq 0}$ are equivalent as $n \rightarrow \infty$ and write $a_{n} \sim b_{n}$ as $n \rightarrow \infty$ if and only if $\lim _{n \rightarrow \infty} \frac{a_{n}}{b_{n}}=1$.

Proposition 3.1. Let $\left(p_{n}\right)_{n \geq 1}$ be a sequence of integers.

1. If $\lim \sup n^{-1} p_{n}<1 / 2$, then $\mu_{n}\left[b_{p_{n}}\left(M_{\ell}\right)\right] \sim\left(\begin{array}{c}n-1 \\ p_{n}\end{array}\right)$ as $n \rightarrow \infty$.

2. If $\lim \inf n^{-1} p_{n}>1 / 2$, then $\mu_{n}\left[b_{p_{n}}\left(M_{\ell}\right)\right] \sim\left(\begin{array}{c}n-1 \\ p_{n}+2\end{array}\right)$ as $n \rightarrow \infty$.

3. If $\lim n^{-1 / 2}\left(p_{n}-n / 2\right)=\alpha$, then $\mu_{n}\left[b_{p_{n}}\left(M_{\ell}\right)\right] \sim \sqrt{\frac{2}{\pi n}} e^{-2 \alpha^{2}} 2^{n-1}$ as $n \rightarrow \infty$.

Applying Proposition 3.1 with a specific choice of the sequence $p_{n}$, we deduce the following corollary. The asymptotic of the binomial coefficient is obtained with Stirling's formula.

Corollary 3.1. Let $p \in(0,1)$ and $p_{n}=[n p]$. Then,

$$
\lim _{n \rightarrow \infty} n^{-1} \log \mu_{n}\left[b_{p_{n}}\left(M_{\ell}\right)\right]=-p \log p-(1-p) \log (1-p)
$$

Proof of Proposition 3.1. From Lemma 2.1, the average Bett numbers is given by

$$
\mu_{n}\left[b_{p_{n}}\left(M_{\ell}\right)\right]=\left(\begin{array}{c}
n-1 \\
p_{n}
\end{array}\right) \mu_{n}\left(\tilde{\tau}>p_{n}\right)+\left(\begin{array}{c}
n-1 \\
p_{n}+2
\end{array}\right) \mu_{n}\left(\tilde{\tau}>n-3-p_{n}\right) .
$$

When $\lim \sup n^{-1} p_{n}<1 / 2$, the weak law of large numbers provided in Lemma 2.3 implies that

$$
\mu_{n}\left(\tilde{\tau}>p_{n}\right) \rightarrow 1 \text { and } \mu_{n}\left(\tilde{\tau}>n-3-p_{n}\right) \rightarrow 0
$$

as $n \rightarrow \infty$, and from large deviations estimates, the convergence speed to zero is exponential. The first point in Proposition 3.1 follows since

$$
\begin{aligned}
b_{p_{n}}\left(n, \mu_{n}\right) & =\left(\begin{array}{c}
n-1 \\
p_{n}
\end{array}\right)\left(\mu_{n}\left(\tilde{\tau}>p_{n}\right)+\frac{\left(n-p_{n}-1\right)\left(n-p_{n}-2\right)}{\left(p_{n}+1\right)\left(p_{n}+2\right)} \mu_{n}\left(\tilde{\tau}>n-3-p_{n}\right)\right) \\
& \sim\left(\begin{array}{c}
n-1 \\
p_{n}
\end{array}\right) .
\end{aligned}
$$

Similarly, when $\lim \inf n^{-1} p_{n}>1 / 2$,

$$
\mu_{n}\left(\tilde{\tau}>p_{n}\right) \rightarrow 0 \text { and } \mu_{n}\left(\tilde{\tau}>n-3-p_{n}\right) \rightarrow 1
$$

as $n \rightarrow \infty$ where the convergence to zero is exponentially fast. The second point in Proposition 3.1 follows.

Finally, in the case $\lim n^{1 / 2}\left(p_{n}-n / 2\right)=\alpha$, the central limit Theorem from Lemma 2.3 yields that as $n \rightarrow \infty$

$$
\mu_{n}\left(\tilde{\tau}>p_{n}\right) \rightarrow 1-F_{\mathcal{N}}\left(\alpha / \sigma_{\tilde{\tau}}\right) \text { and } \mu_{n}\left(\tilde{\tau}>n-3-p_{n}\right) \rightarrow 1-F_{\mathcal{N}}\left(-\alpha / \sigma_{\tilde{\tau}}\right),
$$


where $F_{\mathcal{N}}$ is the repartition function of the standard normal distribution. Furthermore, from the local limit theorem for the binomial distribution,

$$
\left(\begin{array}{c}
n-1 \\
p_{n}
\end{array}\right) \sim\left(\begin{array}{c}
n-1 \\
n-3-p_{n}
\end{array}\right) \sim \sqrt{\frac{2}{\pi n}} e^{-2 \alpha^{2}} 2^{n-1},
$$

as $n \rightarrow \infty$. These estimates yield the last point in Proposition 3.1 since

$$
1-F_{\mathcal{N}}\left(\alpha / \sigma_{\tilde{\tau}}\right)+1-F_{\mathcal{N}}\left(-\alpha / \sigma_{\tilde{\tau}}\right)=1
$$

\subsection{Spatial Polygons}

We perform a similar study in the spatial case. The asymptotic behavior of average highdimensional Betti numbers is given by the following Proposition.

Proposition 3.2. Let $\left(p_{n}\right)_{n \geq 1}$ be a sequence of integers.

1. If $\lim \sup n^{-1} p_{n}<1 / 2$, then $\mu_{n}\left[b_{2 p_{n}}\left(N_{\ell}\right)\right] \sim \sum_{k=0}^{p_{n}}\left(\begin{array}{c}n-1 \\ k\end{array}\right)$ as $n \rightarrow \infty$.

2. If $\lim \inf n^{-1} p_{n}>1 / 2$, then $\mu_{n}\left[b_{2 p_{n}}\left(N_{\ell}\right)\right] \sim \sum_{k=0}^{n-p_{n}-3}\left(\begin{array}{c}n-1 \\ k\end{array}\right)$ as $n \rightarrow \infty$.

3. If $\lim n^{-1 / 2}\left(p_{n}-n / 2\right)=\alpha$, then $\mu_{n}\left[b_{2 p_{n}}\left(N_{\ell}\right)\right] \sim C(\alpha) 2^{n-1}$ as $n \rightarrow \infty$, with

$$
C(\alpha)=\int_{2|\alpha|}^{\infty} \frac{e^{-\frac{u^{2}}{2}}}{\sqrt{2 \pi}} \mathbb{P}\left(|Z|<\frac{u m}{\sigma}\right) \mathrm{d} u,
$$

where $m=\mu(l), \sigma^{2}=\operatorname{Var}(l)$, and $Z$ is standard normal.

Proof of Proposition 3.2. Recall from Lemma 2.2 that the expected Betti number $\mu_{n}\left[b_{2 p}\left(N_{\ell}\right)\right]$ is given by

$$
\mu_{n}\left[b_{2 p}\left(N_{\ell}\right)\right]=2^{n-1}\left(\mu_{n} \otimes \mathcal{B}_{n-1,1 / 2}\right)\left[\mathbf{1}_{\left\{\tau>k ; 0 \leq k \leq p_{n}\right\}}-\mathbf{1}_{\left\{\tau>n-1-k ; 1 \leq k \leq p_{n}+1\right\}}\right]
$$

(we use here the fact that $k$ and $n-1-k$ have the same distribution under $\mathcal{B}_{n-1,1 / 2}$ ). Consider first the case $\lim \sup n^{-1} p_{n}<1 / 2$ and write

$$
\mu_{n}\left(\tau>p_{n}\right) \mathcal{B}_{n-1,1 / 2}\left(0 \leq k \leq p_{n}\right) \leq\left(\mu_{n} \otimes \mathcal{B}_{n-1,1 / 2}\right)\left[\mathbf{1}_{\left\{\tau>k ; 0 \leq k \leq p_{n}\right\}}\right] \leq \mathcal{B}_{n-1,1 / 2}\left(0 \leq k \leq p_{n}\right)
$$

Using the weak law of large numbers $n^{-1} \tau \rightarrow 1 / 2$ under $\mu_{n}$ and the asymptotic for $p_{n}$, we see that $\mu_{n}\left(\tau>p_{n}\right) \rightarrow 1$ as $n \rightarrow \infty$. Hence the equivalent

$$
\left(\mu_{n} \otimes \mathcal{B}_{n-1,1 / 2}\right)\left[\mathbf{1}_{\left\{\tau>k ; 0 \leq k \leq p_{n}\right\}}\right] \sim \mathcal{B}_{n-1,1 / 2}\left(0 \leq k \leq p_{n}\right) .
$$

In the same way,

$\left.0 \leq\left(\mu_{n} \otimes \mathcal{B}_{n-1,1 / 2}\right)\left[\mathbf{1}_{\left\{\tau>n-1-k ; 1 \leq k \leq p_{n}+1\right\}}\right] \leq \mu_{n}\left(\tilde{\tau}>n-1-p_{n}\right) \mathcal{B}_{n-1,1 / 2}\left(1 \leq k \leq p_{n}+1\right)\right)$

and a large deviations argument shows that $\mu_{n}\left(\tau>n-p_{n}\right)$ converges exponentially fast to zero, so that this last term is of smaller order than $\mathcal{B}_{n-1,1 / 2}\left(0 \leq k \leq p_{n}\right)$. This proves the first point. 
Consider now the case $\lim \inf n^{-1} p_{n}>1 / 2$. It appears that many terms cancel out and we have for large $n$

$$
\begin{aligned}
\mu_{n}\left[b_{2 p_{n}}\left(N_{\ell}\right)\right] & =2^{n-1}\left(\mu_{n} \otimes \mathcal{B}_{n-1,1 / 2}\right)\left[\mathbf{1}_{\left\{\tau>k ; 0 \leq k \leq p_{n}\right\}}-\mathbf{1}_{\left\{\tau>k ; n-p_{n}-2 \leq k \leq n-2\right\}}\right] \\
& =2^{n-1}\left(\mu_{n} \otimes \mathcal{B}_{n-1,1 / 2}\right)\left[\mathbf{1}_{\left\{\tau>k ; 0 \leq k \leq n-3-p_{n}\right\}}-\mathbf{1}_{\left\{\tau>k ; p_{n}+1 \leq k \leq n-2\right\}}\right] \\
& \sim \mathcal{B}_{n-1,1 / 2}\left(0 \leq k \leq n-3-p_{n}\right),
\end{aligned}
$$

where the equivalent is proved just as above.

Finally, consider the case $p_{n}=n / 2+\alpha_{n} \sqrt{n}$ with $\alpha_{n} \rightarrow \alpha$. We use the central limit Theorem and write

$$
\begin{aligned}
\mu_{n}\left[b_{2 p_{n}}\left(N_{\ell}\right)\right]= & \left.2^{n-1}\left(\mu_{n} \otimes \mathcal{B}_{n-1,1 / 2}\right)\left[\mathbf{1}_{\left\{\tau>k ; k \leq p_{n}\right\}}-\mathbf{1}_{\left\{\tau>k ; n-p_{n}-2 \leq k \leq n-2\right\}}\right]\right) \\
= & 2^{n-1}\left(\mu_{n} \otimes \mathcal{B}_{n-1,1 / 2}\right)\left[\mathbf{1}_{\left\{n^{-1 / 2}(\tau-n / 2)>n^{-1 / 2}(k-n / 2) ; n^{-1 / 2}(k-n / 2) \leq \alpha_{n}\right\}}\right. \\
& \left.\left.-\mathbf{1}_{\left\{n^{-1 / 2}(\tau-n / 2)>n^{-1 / 2}(k-n / 2) ;-\alpha_{n}-2 n^{-1 / 2} \leq n^{-1 / 2}(k-n / 2) \leq n^{-1 / 2}(n / 2-2)\right\}}\right]\right) \\
\sim & 2^{n-1} \mathbb{E}\left[\mathbf{1}_{\left\{\sigma_{\tau} G_{1}>G_{2} / 2 ; G_{2} / 2<\alpha\right\}}-\mathbf{1}_{\left\{\sigma_{\tau} G_{1}>G_{2} / 2 ; G_{2} / 2>-\alpha\right\}}\right]
\end{aligned}
$$

with $G_{1}$ and $G_{2}$ independent standard Gaussian random variables. The constant $C(\alpha)$ corresponds to the expectation in the last line. Using symetry properties for the distribution of $\left(G_{1}, G_{2}\right)$, we easily verify the announced formula for $C(\alpha)$. This ends the proof of Proposition 3.2.

\section{ASYMPTOTIC BEHAVIOR OF THE POINCARÉ POLYNOMIAL}

\subsection{Planar Polygons}

We will here consider the random Poincaré polynomial $p_{M_{\ell}}(t)$ as given in (2) in the large $n$ limit. We first give a representation of this invariant in terms of random permutations and stopping times.

Lemma 4.1. For any $t>0$, the Poincaré polynomial is given $\mu_{n}$-almost surely by

$$
p_{M_{\ell}}(t)=(1+t)^{n-1}\left(\mathcal{U}_{\Sigma_{n-1}} \otimes \mathcal{B}_{n-1, \frac{t}{1+t}}\right)\left[\mathbf{1}_{\left\{\tau_{\sigma}(\tilde{\ell})>k\right\}}+t^{-2} \mathbf{1}_{\left\{\tau_{\sigma}(\tilde{\ell})>n-1-k\right\}}\right]
$$

As a consequence,

$$
\bar{p}_{M_{\ell}}(t)=(1+t)^{n-1}\left(\mu_{n} \otimes \mathcal{B}_{n-1, \frac{t}{1+t}}\right)\left[\mathbf{1}_{\{\tilde{\tau}>k\}}+t^{-2} \mathbf{1}_{\{\tilde{\tau}>n-1-k\}}\right] .
$$

Thanks to this lemma, we prove the following Proposition giving the asymptotic of the average Poincaré polynomial.

Proposition 4.1. Let $\bar{p}_{M_{\ell}}(t)$ be the mean Poincaré polynomial. When $t>0$,

1. If $0<t<1$, then $\bar{p}_{M_{\ell}}(t) \sim(1+t)^{n-1}$.

2. If $t>1$, then $\bar{p}_{M_{\ell}}(t) \sim(1+t)^{n-1} t^{-2}$.

3. If $t=1$, then the mean total Betti number satisfies $\bar{p}_{M_{\ell}}(1) \sim 2^{n-1}$. 
Proof of Lemma 4.1. Equation (2) together with Lemma 2.1 yield

$$
\begin{aligned}
q(t) & =\sum_{k=0}^{n-1}\left(\begin{array}{c}
n-1 \\
k
\end{array}\right) t^{k} \mathcal{U}_{\Sigma_{n-1}}\left(\tau_{\sigma}(\tilde{\ell})>k\right) \\
& =(1+t)^{n-1} \sum_{k=0}^{n-1}\left(\begin{array}{c}
n-1 \\
k
\end{array}\right)\left(\frac{t}{1+t}\right)^{k}\left(\frac{1}{1+t}\right)^{n-1-k} \mathcal{U}_{\Sigma_{n-1}}\left(\tau_{\sigma}(\tilde{\ell})>k\right) \\
& =(1+t)^{n-1}\left[\left(\mathcal{U}_{\Sigma_{n-1}} \otimes \mathcal{B}_{n-1, \frac{t}{1+t}}\right)\left(\tau_{\sigma}(\tilde{\ell})>k\right)\right] .
\end{aligned}
$$

Please note that in the sum the terms corresponding to $k=n-2$ and $k=n-1$ vanish. Finally, Lemma 4.1 follows from the relation

$$
p_{M_{\ell}}(t)=q(t)+t^{n-3} q\left(t^{-1}\right)+r(t),
$$

with $r(t) \mu_{n}$-almost surely vanishing and from the fact that the distribution of $k$ under $\mathcal{B}_{n-1, \frac{1}{1+t}}$ is equal to the distribution of $n-1-k$ under $\mathcal{B}_{n-1, \frac{t}{1+t}}$.

We use once again the invariance property of $\mu_{n}$ under the action of the symetric group to simplify the expression of the average Poincaré polynomial $\mu_{n}\left[p_{M_{\ell}}(t)\right]$.

Proof of Proposition 4.1. We use the representation of the average Poincaré polynomial given in Lemma 4.1 together with weak convergence for $(\tilde{\tau}, k)$ under $\mu_{n} \otimes \mathcal{B}_{n-1, \frac{t}{1+t}}$ to study the asymptotic behavior.

The weak law of large number for $\tilde{\tau}$ (see Lemma 2.3) and a standard weak law of large numbers for binomial distribution imply that $\left(n^{-1} \tilde{\tau}, n^{-1} k\right)$ converges weakly under $\mu_{n} \otimes \mathcal{B}_{n-1, \frac{t}{1+t}}$ to $\left(0, \frac{t}{1+t}\right)$. The continuous mapping theorem implies that for $0<t<1$ or $t>1$, the following weak convergence holds under $\mu_{n} \otimes \mathcal{B}_{n-1, \frac{t}{1+t}}$ :

$$
\mathbf{1}_{\{\tilde{\tau}>k\}}+t^{-2} \mathbf{1}_{\{\tilde{\tau}>n-1-k\}} \Rightarrow \mathbf{1}_{\left\{\frac{1}{2}>\frac{t}{1+t}\right\}}+t^{-2} \mathbf{1}_{\left\{\frac{1}{2}>1-\frac{t}{1+t}\right\}}=\min \left(1, t^{-2}\right) .
$$

Integrating this (bounded) convergence yield the result for $t \neq 1$.

For $t=1$, the continuous mapping theorem does not hold no longer since the map $(\tilde{\tau}, k) \mapsto \mathbf{1}_{\{\tilde{\tau}>k\}}$ is not continuous at point $(1 / 2,1 / 2)$. We need here the central limit Theorem. From Lemma 2.3 and standard results for binomial distribution, $\left(n^{-1 / 2}(\tilde{\tau}-\right.$ $\left.n / 2), n^{-1 / 2}(k-n / 2)\right)$ converges weakly under $\mu_{n} \otimes \mathcal{B}_{n-1,1 / 2}$ to $\mathcal{N}\left(0, \sigma_{\tilde{\tau}}^{2}\right) \otimes \mathcal{N}(0,1 / 4)$. The continuous mapping Theorem yields

$$
\begin{aligned}
\mathbf{1}_{\{\tilde{\tau}>k\}}+\mathbf{1}_{\{\tilde{\tau}>n-1-k\}} & =\mathbf{1}_{\left\{n^{-1 / 2}(\tilde{\tau}-n / 2)>n^{-1 / 2}(k-n / 2)\right\}}+\mathbf{1}_{\left\{n^{-1 / 2}(\tilde{\tau}-n / 2), n^{-1 / 2}(n / 2-1-k)\right\}} \\
& \Rightarrow \mathbf{1}_{\left\{\sigma_{\tilde{\tau}} G_{1}>G_{2} / 2\right\}}+\mathbf{1}_{\left\{\sigma_{\tilde{\tau}} G_{1}>-G_{2} / 2\right\}}
\end{aligned}
$$

with $G_{1}$ and $G_{2}$ independent standard Gaussian random variables. We integrate this (bounded) convergence and remark that $\mathbb{E}\left(\mathbf{1}_{\left\{\sigma_{\tilde{\tau}} G_{1}>G_{2} / 2\right\}}\right)=\mathbb{E}\left(\mathbf{1}_{\left\{\sigma_{\tilde{\tau}} G_{1}>-G_{2} / 2\right\}}\right)=1 / 2$.

Remark. We can use large deviations results to estimate the speed of convergence in Proposition 4.1 when $t \neq 1$. For example for $0<t<1$, write

$$
\begin{aligned}
\mu_{n}\left[(1+t)^{-(n-1)} p_{M_{\ell}}(t)-1\right] & =\left(\mu_{n} \otimes \mathcal{B}_{n-1, \frac{t}{1+t}}\right)\left[\mathbf{1}_{\{\tilde{\tau}>k\}}-1+t^{-2} \mathbf{1}_{\{\tilde{\tau}>n-1-k\}}\right] \\
& =\left(\mu_{n} \otimes \mathcal{B}_{n-1, \frac{t}{1+t}}\right)\left[\mathbf{1}_{\left\{n^{-1}(\tilde{\tau}-k) \leq 0\right\}}+t^{-2} \mathbf{1}_{\left\{n^{-1}(\tilde{\tau}+k) \geq 1\right\}}\right] .
\end{aligned}
$$


Now large deviations for $n^{-1}(\tilde{\tau}, k)$ under $\left(\mu_{n} \otimes \mathcal{B}_{n-1, \frac{t}{1+t}}\right)$ will give the speed of convergence to 0 in a logarithmic scale.

For $t>1$, we have

$$
\mu_{n}\left[(1+t)^{-(n-1)}\left(p_{M_{\ell}}(t)\right)-t^{-2}\right]=\left(\mu_{n} \otimes \mathcal{B}_{n-1, \frac{t}{1+t}}\right)\left[\mathbf{1}_{\left\{n^{-1}(\tilde{\tau}-k)>0\right\}}+t^{-2} \mathbf{1}_{\left\{n^{-1}(\tilde{\tau}+k)<1\right\}}\right],
$$

and we can use the same method.

\subsection{Spatial Polygons}

We use the same strategy in the spatial case and use formula (6) giving the Poincaré polynomial for generic vector length. Since $\mu$ is diffuse, $\mu_{n}$-almost every vector length is generic and Eq. (6) holds. The related total Betti number is obtained by taking the $t \rightarrow 1$ limit in (6)

$$
\begin{aligned}
p_{N_{\ell}}(1) & =\lim _{t \rightarrow 1} \frac{1}{1-t^{2}}\left(\hat{q}\left(t^{2}\right)-t^{2(n-2)} \hat{q}\left(t^{-2}\right)\right) \\
& =(n-2) \hat{q}(1)-2 \hat{q}^{\prime}(1) \\
& =(n-2) \sum_{j=0}^{n-1} \hat{a}_{j}-2 \sum_{j=0}^{n-1} j \hat{a}_{j}
\end{aligned}
$$

We use the following representations for the Poincaré polynomial:

Lemma 4.2. The Poincaré polynomial is given $\mu_{n}$-almost surely by

$$
p_{N_{\ell}}(t)=\frac{\left(1+t^{2}\right)^{n-1}}{1-t^{2}}\left(\mathcal{U}_{\Sigma_{n-1}} \otimes \mathcal{B}_{n-1, \frac{t^{2}}{1+t^{2}}}\right)\left[\mathbf{1}_{\left\{\tau_{\sigma}(\ell)>k\right\}}-t^{-2} \mathbf{1}_{\left\{\tau_{\sigma}(\ell)>n-k\right\}}\right],
$$

for $0<t<1$ or $t>1$, and by

$$
p_{N_{\ell}}(1)=n 2^{n-1}\left(\mathcal{U}_{\Sigma_{n-1}} \otimes \mathcal{B}_{n-1,1 / 2}\right)\left[\left(\frac{n-2}{n}-\frac{2 k}{n}\right) \mathbf{1}_{\left\{\tau_{\sigma}(\ell)>k\right\}}\right]
$$

for $t=1$.

Proposition 4.2. Let $\bar{p}_{N_{\ell}}$ be the mean Poincaré polynomial associated with random spatial polygons. When $t>0$,

1. If $0<t<1$, then $\bar{p}_{N_{\ell}}(t) \sim \frac{\left(1+t^{2}\right)^{n-1}}{1-t^{2}}$.

2. If $t>1$, then $\bar{p}_{N_{\ell}}(t) \sim \frac{\left(1+t^{2}\right)^{n-1}}{t^{2}\left(t^{2}-1\right)}$.

3. If $t=1$, then the total Betti number satisfies $\bar{p}_{N_{\ell}}(1) \sim n 2^{n-2}$.

Remark. In the case of spatial polygons, the Poincaré polynomial is an even function. Hence its asymptotic mean behavior for $t<0$ follows directly from Proposition 4.2.

Proof of Lemma 4.2. The proof is very similar to the proof of Lemma 4.1. Equation (6) together with Lemma 2.2 yield

$$
\hat{q}(t)=(1+t)^{n-1}\left(\mathcal{U}_{\Sigma_{n-1}} \otimes \mathcal{B}_{n-1, \frac{t}{1+t}}\right)\left[\tau_{\sigma}(\ell)>k\right] .
$$


The case $t \neq 1$ follows from the relation

$$
p_{N_{\ell}}(t)=\frac{1}{1-t^{2}}\left(\hat{q}\left(t^{2}\right)-t^{2(n-2)} \hat{q}\left(t^{-2}\right)\right)
$$

and from the fact that the distribution of $k$ under $\mathcal{B}_{n-1, \frac{1}{1+t^{2}}}$ is equal to the distribution of $n-K$ under $\mathcal{B}_{n-1, \frac{t^{2}}{1+t^{2}}}$.

In the case $t=1$, Eq. (7) and Lemma 2.2 imply

$$
\begin{aligned}
p_{N_{\ell}}(1) & =(n-2) \sum_{j=0}^{n-1} \hat{a}_{j}-2 \sum_{j=0}^{n-1} j \hat{a}_{j} \\
& =n 2^{n-1} \sum_{j=0}^{n-1}\left(\frac{n-2}{n}-\frac{2 j}{n}\right)\left(\begin{array}{c}
n-1 \\
j
\end{array}\right) \mathcal{U}_{\Sigma_{n-1}}\left[\tau_{\sigma}(\ell)>j\right] \\
& =n 2^{n-1}\left(\mathcal{U}_{\Sigma_{n-1}} \otimes \mathcal{B}_{n-1,1 / 2}\right)\left[\left(\frac{n-2}{n}-\frac{2 k}{n}\right) \mathbf{1}_{\left\{\tau_{\sigma}(\ell)>k\right\}}\right]
\end{aligned}
$$

Proof of Proposition 4.2. The case $0<t<1$ and $t>1$ are easily deduced from Lemma 4.2 using the following law of large numbers: under $\mathcal{B}_{n-1, \frac{t^{2}}{1+t^{2}}} \otimes \mathcal{U}_{\Sigma_{n-1}}, n^{-1}(k, \tau)$ converges weakly to $\left(1 / 2, \frac{t^{2}}{1+t^{2}}\right)$ as $n \rightarrow \infty$. Details are omitted since they are as in the proof of Proposition 4.1 .

In the case $t=1$, the central limit Theorem from Lemma 2.3 states that $\left(n^{-1 / 2}(\tilde{\tau}-\right.$ $\left.n / 2), n^{-1 / 2}(k-n / 2)\right)$ converges weakly under $\mu_{n} \otimes \mathcal{B}_{n-1,1 / 2}$ to $\mathcal{N}\left(0, \sigma_{\tilde{\tau}}^{2}\right) \otimes \mathcal{N}(0,1 / 4)$. As a consequence,

$$
\begin{aligned}
n^{-1} 2^{-(n-1)} \mu_{n}\left[p_{N_{\ell}}(1)\right] & =\left(\mu_{n} \otimes \mathcal{B}_{n-1,1 / 2}\right)\left[\left(\frac{n-2}{n}-\frac{2 k}{n}\right) \mathbf{1}_{\{\tau>k\}}\right] \\
& \rightarrow \mathbb{E}\left[1-2 \frac{1}{2} \mathbf{1}_{\left\{\sigma_{\tau} G_{1}>G_{2} / 2\right\}}\right]=\frac{1}{2},
\end{aligned}
$$

whith $G_{1}$ and $G_{2}$ independent standard Gaussian random variables.

Remark. In order to estimate the speed of convergence in Proposition 4.2 when $t \neq 1$, we can use for $0<t<1$ the expression

$\mu_{n}\left[\left(1-t^{2}\right)\left(1+t^{2}\right)^{-(n-1)} p_{M_{\ell}}(t)-1\right]=\left(\mu_{n} \otimes \mathcal{B}_{n-1, \frac{t^{2}}{1+t^{2}}}\right)\left[\mathbf{1}_{\left\{n^{-1}(\tau-k) \leq 0\right\}}-t^{-2} \mathbf{1}_{\left\{n^{-1}(\tau+k)>1\right\}}\right]$

and for $t>1$

$\mu_{n}\left[\left(t^{2}-1\right)\left(1+t^{2}\right)^{-(n-1)} p_{M_{\ell}}(t)-t^{-2}\right]=\left(\mu_{n} \otimes \mathcal{B}_{n-1, \frac{t^{2}}{1+t^{2}}}\right)\left[\mathbf{1}_{\left\{n^{-1}(\tau-k)>0\right\}}-t^{-2} \mathbf{1}_{\left\{n^{-1}(\tau+k) \leq 1\right\}}\right]$.

Large deviations results for $n^{-1}(\tau, k)$ under $\left(\mu_{n} \otimes \mathcal{B}_{n-1, \frac{t^{2}}{1+t^{2}}}\right)$ would give the speed of convergence in a logarithmic scale. 


\subsection{Higher Moments}

We consider here the higher moments of the Poincaré polynomial and prove that their asymptotic behavior is given by the first moment. To this aim, we prove a weak law of large numbers for the renormalized Poincaré polynomial.

We begin with the case of planar polygon.

Proposition 4.3. For any $t>0$, the following weak convergence holds under $\mu_{n}$ as $n \rightarrow \infty$

$$
(1+t)^{-(n-1)} p_{M_{\ell}(t)} \Rightarrow \min \left(1, t^{-2}\right) .
$$

As a consequence, for any $t>0$ and $v \in \mathbb{N}$,

$$
\mu_{n}\left[p_{M_{\ell}}(t)^{v}\right] \sim\left(\mu_{n}\left[p_{M_{\ell}}(t)\right]\right)^{v}
$$

Proof of Proposition 4.3. Proposition 4.1 states that the expectation under $\mu_{n}$ of $(1+$ $t)^{-(n-1)} p_{M_{\ell}(t)}$ converges to $\min \left(1, t^{-2}\right)$ as $n \rightarrow \infty$. Hence, weak convergence will be proved as soon as we show that the variance under $\mu_{n}$ of $(1+t)^{-(n-1)} p_{M_{\ell}(t)}$ goes to zero. We use the representation of the Poincaré polynomial from Lemma 4.1 and the replica trick to compute the second moment

$$
\mu_{n}\left[(1+t)^{-2(n-1)} p_{M_{\ell}}(t)^{2}\right]=\left(\mu_{n} \otimes \mathcal{B}_{n-1, \frac{t}{1+t}}^{\otimes 2} \otimes \mathcal{U}_{\Sigma_{n-1}}^{\otimes 2}\right)[\operatorname{Prod}] .
$$

with

$$
\operatorname{Prod}=\left(\mathbf{1}_{\left\{\tau_{\sigma_{1}}(\tilde{\ell})>k_{1}\right\}}+t^{-2} \mathbf{1}_{\left\{\tau_{\sigma_{1}}(\tilde{\ell})>n-1-k_{1}\right\}}\right)\left(\mathbf{1}_{\left\{\tau_{\sigma_{2}}(\tilde{\ell})>k_{2}\right\}}+t^{-2} \mathbf{1}_{\left\{\tau_{\sigma_{2}}(\tilde{\ell})>n-1-k_{2}\right\}}\right) .
$$

We need to show that the two factors of Prod are asymptotically independent in the limit $n \rightarrow \infty$. This would yield

$$
\mu_{n}\left[(1+t)^{-2(n-1)} p_{M_{\ell}}(t)^{2}\right] \sim\left(\mu_{n}\left[(1+t)^{-(n-1)} p_{M_{\ell}}(t)\right]\right)^{2},
$$

and hence the variance of $\frac{p_{M_{\ell}}(t)}{(1+t)^{n-1}}$ would converge to zero as $n \rightarrow \infty$. We now prove asymptotic independence of the two factors. When $0<t<1$ or $t>1$ the aymptotic independence follows from the weak law of large numbers obtained in Lemma 2.3, both factors converging weakly to $\min \left(1, t^{-2}\right)$ (note that the distribution of $\tau_{\sigma}(\tilde{\ell})$ under $\mu_{n} \otimes \mathcal{U}_{\Sigma_{n-1}}$ is equal to the distribution of $\tilde{\tau}(\ell)$ under $\mu_{n}$ ). When $t=1$, we use the bivariate central limit Theorem stated in Lemma 4.3 in the Appendix. Weak convergence is proved.

The convergence of the moments is a direct consequence of the weak convergence once we remark that the renormalized Poincare polynomial $(1+t)^{-(n-1)} p_{M_{\ell}}(t)$ is $\mu_{n}$ almost surely bounded by $1+t^{-2}$ (this is clear from the representation given in Lemma 4.1).

We consider now the higher moments of the Poincaré polynomial for spatial polygons spaces. The results and methods are very similar to one of the planar case and are based on Lemma 4.2. Hence we give only the main lines of the proof. 
Proposition 4.4. The following weak convergence holds under $\mu_{n}$ as $n \rightarrow \infty$,

$$
\begin{aligned}
& \text { if } 0<t<1, \quad\left(1+t^{2}\right)^{-(n-1)} p_{N_{\ell}}(t) \Rightarrow\left(1-t^{2}\right)^{-1}, \\
& \text { if } t>1, \quad\left(1+t^{2}\right)^{-(n-1)} p_{N_{\ell}}(t) \Rightarrow t^{-2}\left(t^{2}-1\right)^{-1}, \\
& \text { if } t=1, \quad n^{-1} 2^{-n} p_{N_{\ell}}(1) \Rightarrow 1 / 4 .
\end{aligned}
$$

As a consequence, for any $t>0$ and $v \in \mathbb{N}$,

$$
\mu_{n}\left[p_{N_{\ell}}(t)^{\nu}\right] \sim\left(\mu_{n}\left[p_{N_{\ell}}(t)\right]\right)^{v} .
$$

Proof of Proposition 4.4. The proof is similar to the proof of Proposition 4.3 with the following expression of the renormalized Poincaré polynomial deduced from Lemma 2.2: for $0<t<1$ or $t>1$

$$
\left(1-t^{2}\right)\left(1+t^{2}\right)^{-(n-1)} p_{N_{\ell}}(t)=\left(\mathcal{U}_{\Sigma_{n-1}} \otimes \mathcal{B}_{n-1, \frac{t^{2}}{1+t^{2}}}\right)\left[\mathbf{1}_{\left\{\tau_{\sigma}(\ell)>k\right\}}-t^{-2} \mathbf{1}_{\left\{\tau_{\sigma}(\ell)>n-k\right\}}\right],
$$

and for $t=1$

$$
n^{-1} 2^{-(n-1)} p_{N_{\ell}}(1)=\left(\mathcal{U}_{\Sigma_{n-1}} \otimes \mathcal{B}_{n-1,1 / 2}\right)\left[\left(\frac{n-2}{n}-\frac{2 k}{n}\right) \mathbf{1}_{\left\{\tau_{\sigma}(\ell)>k\right\}}\right]
$$

Convergence of the expectation was proved in Proposition 4.2. The variance is computed using thanks to the replica trick and is shown to converge to zero because of the asymptotic independence of $\mathbf{1}_{\left\{\tau_{\sigma_{i}}(\ell)>k_{i}\right\}}, i=1,2$ under $\mu_{n} \otimes \mathcal{B}_{n-1, \frac{t^{2}}{1+t^{2}}}^{\otimes 2} \otimes \mathcal{U}_{\Sigma_{n-1}}^{\otimes 2}$ (see Lemma 4.3).

\section{APPENDIX}

Proof of Lemma 2.3. The weak law of large number is a consequence of the central limit theorem that we prove now. Let $p_{n}=\frac{n}{2}+\alpha_{n} \sqrt{n}$ with $\alpha_{n} \rightarrow \alpha$ as $n \rightarrow \infty$. Using the definition of $\tilde{\tau}$,

$$
\begin{aligned}
\mu_{n}\left(\tilde{\tau} \leq p_{n}\right) & =\mu_{n}\left(\tilde{l}_{n}+\sum_{i=1}^{p_{n}} \tilde{l}_{i}-\sum_{i=p_{n}+1}^{n-1} \tilde{l}_{i} \geq 0\right) \\
& =\mu_{n}\left(n^{-1 / 2} \tilde{l}_{n}+n^{-1 / 2}\left(\sum_{i=1}^{p_{n}} \tilde{l}_{i}-\sum_{i=p_{n}+1}^{n-1} \tilde{l}_{i}\right) \geq 0\right) .
\end{aligned}
$$

We now prove that $n^{-1 / 2} \tilde{l}_{n}$ converges weakly to zero and that $n^{-1 / 2}\left(\sum_{i=1}^{p_{n}} \tilde{l}_{i}-\sum_{i=p_{n}+1}^{n-1} \tilde{l}_{i}\right)$ satisfies a central limit theorem. To see this, we denote by $F_{\mu}$ the repartition function of $\mu$, and remark that the distribution of $\tilde{l}_{n}$ is given by

$$
\mu_{n}\left(\tilde{l}_{n} \leq x\right)=F_{\mu}(x)^{n},
$$

so that

$$
\mu_{n}\left(n^{-1 / 2} \tilde{l}_{n}>\varepsilon\right)=1-\left(F_{\mu}\left(\varepsilon n^{1 / 2}\right)\right)^{n}
$$


The exponential Markov inequality implies

$$
1-F_{\mu}\left(\varepsilon n^{1 / 2}\right) \leq \exp \left(-\eta \varepsilon n^{1 / 2}\right) \int e^{\eta x} \mu(\mathrm{d} x), \eta>0 .
$$

As a consequence, for large $n$

$$
\begin{aligned}
\mu_{n}\left(n^{-1 / 2} \tilde{l}_{n}>\varepsilon\right) & \leq 1-\left(1-\exp \left(-\eta \varepsilon n^{1 / 2}\right) \int e^{\eta x} \mu(\mathrm{d} x)\right)^{n} \\
& \rightarrow 0 \text { as } n \rightarrow \infty
\end{aligned}
$$

This implies the weak convergence $n^{-1 / 2} \tilde{l}_{n}$ to zero. Conditionally to $\tilde{l}_{n}=u$, the other components $\left(l_{i}\right)_{1 \leq i \leq n-1}$ are i.i.d. with conditional distribution given by

$$
\mu_{n}\left(\tilde{l}_{i} \leq x \mid \tilde{l}_{n}=u\right)=\frac{F_{\mu}(x \wedge u)}{F_{\mu}(u)} .
$$

Denote by $m(u)$ and $\sigma^{2}(u)$ the related conditional expectation and variance. From the central limit theorem for independent variables, conditionally to $\tilde{l}_{n}=u$, the quantity $n^{-1 / 2}\left(\sum_{i=1}^{p_{n}} \tilde{l}_{i}-\right.$ $\left.\sum_{i=p_{n}+1}^{n-1} \tilde{l}_{i}\right)$ converges weakly to a Gaussian distribution of mean $2 \alpha m(u)$ and variance $\sigma^{2}(u)$. Hence the conditional probability

$$
\mu_{n}\left[n^{-1 / 2}\left(\sum_{i=1}^{p_{n}} \tilde{l}_{i}-\sum_{i=p_{n}+1}^{n-1} \tilde{l}_{i}\right) \geq 0 \mid \tilde{l}_{n}=u\right]
$$

converges to $F_{\mathcal{N}}(2 \alpha m(u) / \sigma(u))$ as $n \rightarrow \infty$. We now have to integrate this with respect to $\tilde{l}_{n}$. Taking into account that $\tilde{l}_{n}$ converges weakly to $l_{\max }=\inf \left\{x \in \mathbb{R} ; F_{\mu}(x)=1\right\} \in(0,+\infty]$ as $n \rightarrow \infty$ and that $(m(u), \sigma(u)) \rightarrow(m, \sigma)$ as $u \rightarrow l_{\max }$, we see that

$$
\mu_{n}\left[n^{-1 / 2}\left(\sum_{i=1}^{p_{n}} \tilde{l}_{i}-\sum_{i=p_{n}+1}^{n-1} \tilde{l}_{i}\right) \geq 0\right] \rightarrow F_{\mathcal{N}}(2 \alpha m / \sigma)
$$

This proves the central limit theorem for $\tilde{\tau}$.

We now prove the large deviation estimate. Since

$$
\left.\mu_{n}(\tilde{\tau} \leq(1 / 2-\varepsilon) n)\right)=\mu_{n}\left(\tilde{l}_{n}+\sum_{i=1}^{[(1 / 2-\varepsilon) n]} \tilde{l}_{i}-\sum_{i=[(1 / 2-\varepsilon) n]}^{n-1} \tilde{l}_{i} \geq 0\right)
$$

we will provide large deviations estimates for the random sum

$$
S_{n}=\tilde{l}_{n}+\sum_{i=1}^{[(1 / 2-\varepsilon) n]} \tilde{l}_{i}-\sum_{i=[(1 / 2+\varepsilon) n]}^{n-1} \tilde{l}_{i}
$$

For $t \in \mathbb{R}$, the logarithmic moment generating function is defined by

$$
\Lambda_{n}(t)=\log \left(\mu_{n}\left(\exp \left(t S_{n}\right)\right)\right) \text {. }
$$


Using Laplace method, we see that as $n \rightarrow \infty, n^{-1} \Lambda_{n}(t)$ converges to

$$
\Lambda(t)=(1 / 2-\varepsilon) \int e^{t y} \mu(\mathrm{d} y)+(1 / 2+\varepsilon) \int e^{t y} \mu(\mathrm{d} y) .
$$

Using Gärtner-Ellis theorem, see e.g. [1], we deduce a large deviations principle for the sum $n^{-1} S_{n}$ of speed $n$ and of good rate function $I$ being the Fenchel-Legendre transform of $\Lambda$. The exact form of $I$ is irrelevant here but it is important to see that $I$ is strictly positive on $[0, \infty)$. Standard arguments from large deviations theory (see [1]) give that $I$ vanishes only at $(1 / 2-\varepsilon) m-(1 / 2+\varepsilon) m=-2 \varepsilon m<0$, and hence the action $I$ is negative on $[0, \infty)$. As a consequence, the large deviations principle states that

$$
\lim \sup n^{-1} \log \mu_{n}(\tilde{\tau} \leq(1 / 2-\varepsilon) n) \leq-\inf _{[0, \infty)} I<0 .
$$

The same technique is used to deal with $\mu_{n}(\tilde{\tau} \geq(1 / 2+\varepsilon) n)$ and this proves the Lemma.

Lemma 4.3. The following bivariate Central Limit Theorem holds under $\mu_{n} \otimes \mathcal{U}_{\Sigma_{n-1}}^{\otimes 2}$ :

$$
n^{-1 / 2}\left(\tau_{\sigma_{1}}(\ell)-n / 2, \tau_{\sigma_{2}}(\ell)-n / 2\right) \Rightarrow \mathcal{N}\left(0, \sigma_{\tau}^{2}\right)^{\otimes 2}
$$

It also holds for $\tilde{\tau}$

Proof of Lemma. The proof needs a bivariate central limit Theorem for $\left(\tau_{\sigma_{1}}(\tilde{\ell}), \tau_{\sigma_{2}}(\tilde{\ell})\right)$ under Let $p_{n, i}=\frac{n}{2}+\alpha_{n, i} \sqrt{n}$ with $\alpha_{n, i} \rightarrow \alpha$ as $n \rightarrow \infty$ for $i=1,2$. By the definition of $\tilde{\tau}_{\sigma_{i}}$,

$$
\begin{aligned}
\left(\mu_{n} \otimes \mathcal{U}_{\Sigma_{n-1}}^{\otimes 2}\right) & \left(\tilde{\tau}_{\sigma_{i}} \leq p_{n, i} ; i=1 . .2\right) \\
& =\left(\mu_{n} \otimes \mathcal{U}_{\Sigma_{n-1}}^{\otimes 2}\right)\left(n^{-1 / 2} \tilde{l}_{n}+n^{-1 / 2}\left(\sum_{j=1}^{p_{n, i}} \tilde{l}_{\sigma_{i}(j)}-\sum_{j=p_{n, i}+1}^{n-1} \tilde{l}_{\sigma(j)}\right) \geq 0 ; i=1,2\right) .
\end{aligned}
$$

We know from the proof of Lemma 2.3 that $n^{-1 / 2} \tilde{l}_{n}$ converges weakly to zero. It remains to check that $n^{-1 / 2}\left(\sum_{j=1}^{p_{n, i}} \tilde{l}_{\sigma_{i}(j)}-\sum_{j=p_{n, i}+1}^{n-1} \tilde{l}_{\sigma_{i}(j)}\right)_{i=1,2}$ satisfies a bivariate central limit theorem. Let $\theta_{i}, i=1,2$ be real numbers, and consider the linear combination

$$
\sum_{i=1}^{2} \theta_{i} n^{-1 / 2}\left(\sum_{j=1}^{p_{n, i}} \tilde{l}_{\sigma_{i}(j)}-\sum_{j=p_{n, i}+1}^{n-1} \tilde{l}_{\sigma_{i}(j)}=n^{-1 / 2} \sum_{j=1}^{n-1}\left(\theta_{1} \varepsilon_{n, 1}(j)+\theta_{1} \varepsilon_{n, 2}(j)\right) \tilde{l}_{j},\right.
$$

where we set $\varepsilon_{n, i}(j)=2 \mathbf{1}_{\left\{\sigma_{i}(j) \leq p_{n, i}\right\}}-1$. Conditionally to $\tilde{l}_{n}=u$, the components $\tilde{l}_{j}$ are i.i.d. with mean $m(u)$ and variance $\sigma(u)$, and hence the above sum is a linear triangular array of independent variables with random coefficients $\left(\theta_{1} \varepsilon_{n, 1}(j)+\theta_{1} \varepsilon_{n, 2}(j)\right)_{1 \leq j \leq n-1}$. The coefficients are almost surely bounded and satisfy a weak law of large numbers under $\mathcal{U}_{\Sigma_{n-1}}^{\otimes 2}$

$$
n^{-1} \sum_{j=1}^{n-1}\left(\theta_{1} \varepsilon_{n, 1}(j)+\theta_{1} \varepsilon_{n, 2}(j)\right)^{2} \rightarrow \theta_{1}^{2}+\theta_{2}^{2}
$$

(note that the empirical distribution $\frac{1}{n-1} \sum_{j=1}^{n-1} \delta_{\left(\varepsilon_{n, 1}(j), \varepsilon_{n, 1}(j) \varepsilon_{n, 2}(j)\right)}$ converges weakly to the uniform distribution on $\{( \pm 1, \pm 1)\})$. As a consequence, conditionally to $\tilde{l}_{n}=u$, the above 
sum converges to a Gaussian random variables of mean $2\left(\alpha_{1} \theta_{1}+\alpha_{2} \theta_{2}\right) m(u)$ and variance $\left(\theta_{1}^{2}+\theta_{2}^{2}\right) \sigma^{2}(u)$. Integrating with respect to $\tilde{l}_{n}$ we obtain that the sum converges weakly to a Gaussian random variables with mean $2\left(\alpha_{1} \theta_{1}+\alpha_{2} \theta_{2}\right) m$ and variance $\left(\theta_{1}^{2}+\theta_{2}^{2}\right) \sigma^{2}$. This proves the bivariate central limit theorem with asymptotic independent components.

\section{REFERENCES}

[1] A. Dembo and O. Zeitouni, Large deviations techniques and applications, Vol. 38, Applications of mathematics, stochastic modelling and applied probability, 2nd edition, Springer, New york, 1998.

[2] M. Farber, Topology of random linkages, Algebraic Geometric Topol 8 (2008), 155-172.

[3] M. Farber, Topology of robot motion planning, In: P. Biran, O. Cornea, and F. Lalonde, editors, Morse theoretic methods on nonlinear analysis and in symplectic topology, Nato Science Series, Springer-Netherlands, Vol. 217, 2006, pp. 185-230.

[4] M. Farber and T. Kappeler, Betti numbers of random manifolds, Homol, Homotopy Appl 10 (2007), 205-222.

[5] M. Farber and D. Schütz, Homology of planar polygon spaces, Geometry Dedicata 25 (2007), $75-92$.

[6] J. C. Hausmann, Sur la topologie des bras articulés, In Algebraic topolgy Poznan 1989, Lecture notes in mathematics, Springer-Verlag, 1474 (1989), 146-160.

[7] J. C. Hausmann and A. Knutson, Cohomology rings of polygon spaces, Ann Inst Fourier 48 (1998), 281-321.

[8] A. Klyachko, Spatial polygons and configurations of points in the projective line, Algebraic geometry and its applications, Aspects Math E25 (1994), 67-84.

[9] H. Lebesgue, Leçons sur les constructions géométriques, professées au Collège de France en 1940-1941, Gauthier-Villars, Paris, 1950.

[10] W. Thurston and J. Weeks, The mathematics of the three-dimensional manifolds, Scientific American 251 (1984), 108-120. 